\title{
Conscious State
}

National Cancer Institute

\section{Source}

National Cancer Institute. Conscious State. NCI Thesaurus. Code C88434.

A level of awareness that can be described as being alert. 